\title{
O ESTUDO DO DESENVOLVIMENTO REGIONAL: UMA ANÁLISE DA PRODUÇÃO CIENTÍFICA INTERNACIONAL E DOS "HOT-TOPICS"
}

\author{
REGIONAL DEVELOPMENT: AN ANALYSIS OF SCIENTIFIC INTERNATIONAL PRODUCTION \\ AND "HOT-TOPICS"
}

\section{Thiago Reis Xavier \\ Doutorando do Programa de Pós-Graduação em Administração da Universidade Federal de Santa Maria - Santa Marina (RS), Brasil.}

\section{Raoni de Oliveira Inácio}

Mestre em administração do Programa de Pós-Graduação em Administração da Universidade Federal de Santa Maria - Santa Marina (RS), Brasil.

\section{Milton Luiz Wittmann}

Professor Associado IV da Universidade Federal de Santa Maria -

Santa Marina (RS), Brasil.

\section{Juliana Kern}

Graduanda do curso de Administração da Universidade

Federal de Santa Maria - Santa Maria (RS), Brasil.
Data de recebimento: 24-01-2013 Data de aceite: 04-10-2013

\section{RESUMO}

Este estudo tem como objetivo realizar um levantamento sobre as publicações acerca do desenvolvimento regional no período de 2001 a 2011. Além de procurar identificar hot-topics, ou seja, assuntos comumente relacionados à temática central do estudo. A coleta de dados ocorreu na base ISI Web of Science e a análise se baseou nos cálculos dos índices $h$-b e $m$. Os resultados apresentam um progressivo aumento do número de publicações a partir de 2007. O idioma inglês é o predominante, sendo os Estados Unidos a origem da maior quantidade de estudos. Dentre os temas considerados hot-topics estão: economy, strategy, networks, innovation, cluster, globalization, institution e governance.

Palavras-chave: análise bibliométrica; desenvolvimento regional; desenvolvimento territorial; desenvolvimento local.

\section{ABSTRACT}

This bibliometric study conducts a lifting on publications about regional development between 2001 and 2011. Data collection occurred at the base of the ISI Web of Science. In the results, we identified 10 indices on the subject, besides the "hot-topics" related administration. The analysis was based on calculations of the $h-b$ index and $m$ index. The results show a progressive increase in number of publications since 2007. The English is the predominant language, with the United States being the source of the largest number of studies. Among the topics considered "hot-topics" are: economy, strategy, networks, innovation, cluster, globalization, institution e governance.

Keywords: bibliometric analysis; regional development; territorial development; local development.

Milton Luiz Wittmann

wittmann@profwittmann.com

Juliana Kern

julianakern@ymail.com 


\section{INTRODUÇÃO}

O entendimento do conceito de desenvolvimento necessita ser compreendido a partir de uma perspectiva sistêmica e multidisciplinar, que envolva aspectos sociais, culturais, ecológicos, econômicos e espaciais. Multidisciplinaridade que torna o tema objeto de especulação por parte dos pesquisadores e profissionais de diversas áreas (SACHS, 1986).

Nesses preceitos, Dallabrida (2000) compreende que o processo de desenvolvimento regional compreende o crescente esforço das sociedades locais na formulação de políticas territoriais com o intuito de discutir questões centrais da complexidade contemporânea, o que torna a região o sujeito de seu próprio processo de desenvolvimento.

O entendimento do desenvolvimento regional não está atrelado apenas ao crescimento econômico, mas também a fatores sociais, culturais, ambientais e políticos (CAIDEN; CARAVANTES, 2004), acompanhado da melhoria na qualidade de vida (OLIVEIRA, 2002), isso inclui alterações da composição da sociedade e a alocação de recursos pelos diferentes setores da economia, de forma a melhorar os indicadores de bem-estar econômico e social (pobreza, desemprego, desigualdade, condições de saúde, alimentação, educação e moradia) (VASCONCELLOS; GARCIA, 1998).

Considerando os pressupostos apresentados e orientando-se pela emergência e importância de estudos relacionados à temática do desenvolvimento regional, o presente estudo tem como objetivo realizar um levantamento sobre as publicações acerca do desenvolvimento regional no período de 2001 a 2011. Além de procurar identificar hot-topics, ou seja, assuntos comumente relacionados à temática central do estudo.

Os hot-topics foram anteriormente utilizados por Madruga e Silva (2008) para analisar publicações sobre o tema sustentabilidade no banco de dados da ISI Web of Science. Procedimento também adotado por Velter et al. (2010), que aprofundaram conhecimentos sobre as publicações na área da sustentabilidade e identificaram quais tópicos relacionados ao tema são considerados hot-topics no período entre 2000 e 2010.
Com a utilização dos instrumentos de análise utilizados pelos autores acima, pretende-se promover um estudo no qual foram coletados os dados através de uma busca simultânea dos termos desenvolvimento regional (regional development), desenvolvimento territorial (territorial development) e desenvolvimento local (local development) na base de periódicos ISI Web of Science.

O artigo, além desta introdução, está estruturado em mais cinco seções: revisão sobre o estudo do desenvolvimento regional, onde buscou identificar a evolução de seu conceito e compreensões acerca do tema; método de pesquisa; análise dos resultados; considerações finais, além das referências bibliográficas.

\section{DESENVOLVIMENTO REGIONAL: ALGUNS PRESSUPOSTOS TEÓRICOS}

Para que se possa iniciar uma discussão sobre o tema desenvolvimento regional (local, territorial), faz-se necessário compreender separadamente os conceitos de região e de desenvolvimento. No que se refere ao primeiro termo, Bassan e Siedenberg (2008) ressaltam que uma região é representada por características relacionadas à (1) formação geomorfológica, econômicosocial, histórica e cultural, (2) distribuição espacial da população, (3) origens do processo produtivo, (4) base econômica local, (5) aspectos políticos e (6) aspectos administrativos.

Sob forma flexiva e complexa, a região não se limita ao espaço físico das relações, situando-se em um espaço cibernético representado por relações virtuais. O que a leva a adquirir um caráter pós-moderno virtual e imaginário, no qual os cidadãos jamais conhecerão a maioria dos seus corregionais. Mas, que, independente desse fato, fazem parte de uma mesma comunidade (BOISIER, 1999).

Por outro lado, evidenciando aspectos relacionados ao desenvolvimento, Dallabrida (2000) ressalta que esse não supõe apenas o crescimento econômico. Visão compartilhada por Oliveira (2002), que compreende o desenvolvimento como atrelado a fatores sociais, culturais e ambientais acompanhados da melhoria na qualidade de vida, ou seja, inclui alterações sociais e alocação de recursos pelos diferentes setores da 
economia, de forma a melhorar os indicadores de bem-estar econômico e social (pobreza, desemprego, desigualdade, condições de saúde, alimentação, educação e moradia) (VASCONCELLOS; GARCIA, 1998).

Para Sachs (1986), o entendimento do conceito de desenvolvimento necessita ser compreendido a partir de uma perspectiva sistêmica e multidisciplinar, que envolva aspectos sociais, culturais, ecológicos, econômicos e espaciais. O que torna mais fácil compreender o significado do desenvolvimento regional, que conforme Boisier (1996, p. 33) resulta de "um processo de mudança social sustentada que tem como finalidade última o progresso permanente da região, da comunidade regional como um todo e de cada indivíduo residente nela".

A perspectiva acerca do desenvolvimento regional, apresentada por Boisier (1996), Vasconcellos e Garcia (1998), Dallabrida (2000) Vázquez Barquero (2001) e Basssan e Siedenberg (2008), passou a ser mais amplamente discutida no decorrer das últimas décadas. Especialmente quando uma abordagem formada por políticas propostas e administradas pelos governos locais e regionais (que procuram assegurar o desenvolvimento econômico de cidades e regiões de "baixo para cima") passou a ganhar força (em detrimento às chamadas políticas de "cima para baixo"). Nesse panorama, as comunidades regionais passaram a atuar no sentido de tornar mais eficientes seus sistemas (BOISIER, 1996).

Vázquez Barquero (2001) lembra que até a década de 1970, as políticas de desenvolvimento regional encontravam-se centradas na atração de empresas externas para as regiões periféricas. Este cenário ocorreu, sobretudo, através de subvenções, auxílios, incentivos, investimentos públicos em infraestrutura com o intuito de formar polos de crescimento, essencialmente baseados por modelos exógenos de desenvolvimento regional.

No entanto, mesmo diante do sucesso de determinados casos de desenvolvimento regional baseados nesse modelo exógeno, percebeu-se que, nem sempre, as políticas de desenvolvimento, baseadas na atração de empresas externas, atingiram seu objetivo (VÁZQUEZ BARQUERO, 2001). O que estimulou o surgimento, a partir dos anos 1980, na Europa, uma concepção que valorizava o local como referência territorial (sentido de lugar).

Segundo o Gobierno Vasco (1994), essa concepção surgiu com o intuito de aproximar as pessoas, apoiar a solidariedade comunitária e instrumentalizar a comunidade, envolvendo-a efetivamente na superação dos problemas e na promoção do desenvolvimento sob uma perspectiva endógena.

Através da perspectiva endógena, o desenvolvimento regional passou a ser discutido, a partir dos anos 1990, sob a ótica de políticas descentralizadas. Tal cenário promoveu a emergência de modelos baseados em pequenas empresas situadas em regiões consideradas "periféricas", que passaram a ser analisadas a partir de uma visão integradora composta por três esferas: (1) a econômica (relação entre as empresas), (2) a social (as características da estrutura social e as condições para a coesão social) e (3) a territorial (organização do território e a estrutura de governança no plano local) (TAPIA, 2005).

A promoção de ações endógenas fez que localmente as instituições de apoio competitivo e as empresas privadas ganhassem novas missões e definições de seus negócios, ligadas ao desenvolvimento regional (PIRES, 2001). Corroborando com essa perspectiva, Martins (2002) ressalta que o desenvolvimento regional não se trata apenas de buscar o atendimento às carências materiais, mas causar a identificação e a promoção das qualidades, capacidades e competências locais. Em um contexto no qual as pessoas devem participar ativamente e não apenas serem beneficiárias do desenvolvimento.

O que se pode extrair desses modelos endógenos de desenvolvimento regional é que a articulação dos diversos atores representativos de uma localidade é fundamental para o desenvolvimento local (LORENZO; FONSECA, 2008). Esses atores são representados pela sociedade civil, as organizações não governamentais, as instituições privadas e o próprio governo. Cada um dos atores tem seu papel para contribuir com o desenvolvimento local (BUARQUE, 2004). A concepção de local como ponto de partida da temática de desenvolvimento, pode ser justificada por razões das características globalizadas, que promove a reafirmação do local e a importância da diferenciação dos lugares (ALBAGLI, 1999). 


\section{MÉTODO DE ESTUDO}

Visando a alcançar os objetivos propostos, foi realizada uma pesquisa bibliométrica, a qual constitui em uma técnica de medição dos índices de produção e disseminação do conhecimento científico (FONSECA, 1977, 1986).

Sobre esse tipo de pesquisa, Araújo (2006) ressalta que sua área mais importante da bibliometria é a análise de citações, que contribui para o desenvolvimento da ciência, possibilitando que um pesquisador seja devidamente reconhecido pelos seus pares. Além de apontar literaturas relevantes e atualizadas para o desenvolvimento de trabalhos científicos.

Com relação às análises, ressalta-se que as mesmas sustentaram-se em abordagens de natureza quantitativa e qualitativa. Nas análises quantitativas foram caracterizadas as seguintes variáveis: (a) total de publicações; (b) autores com maior número de publicações; (c) áreas temáticas; (d) título das fontes; (e) ano das publicações; (f) instituições; (g) agências de financiamento; (h) idiomas e (i) países. As informações quantitativas permitiram a realização da fase qualitativa, que analisou os assuntos tratados nas publicações pesquisadas, tendo como foco o conteúdo das mesmas, as palavras-chave e a relevância das temáticas.

Posteriormente, buscando contemplar o objetivo de averiguar os assuntos de maior relevância relacionados à temática proposta (hot-topics), analisou-se o número de vezes que cada publicação foi citada através do índice $h-b$ e do índice $m$.

O índice h-b é apresentado por Banks (2006) como uma extensão do h-index, apresentado por Hirsch (2005) em sua pesquisa denominada "An index to quantify an individual's scientific research output", que tem como função quantificar o impacto e relevância da produção científica individual.

As contribuições fornecidas pelo h-index permitiram Banks (2006) a chegar ao índice $h$-b que pode ser obtido através do número de citações de um tópico ou combinação em um período estabelecido, listados em ordem decrescente de citações. O mesmo pode ser encontrado em publicações que tenham obtido um número de citações igual ou maior à sua posição no ranking.
Os estudos de Banks (2006) também apresentam o índice $m$, obtido através da divisão do índice $h-b$ pelo período de anos que se deseja obter informações $(n)$. Para análise do índice $m$ devem ser levadas em conta as seguintes considerações:

- $0<m \leq 0,5$ : o tópico/combinação pode ser de interesse para pesquisadores em um campo específico de pesquisa, onde o campo engloba uma comunidade pequena.

- 0,5<m $\leq 2$ : o tópico/combinação provavelmente pode ser um hot-topic como área de pesquisa, onde a comunidade é muito grande ou o tópico/combinação apresenta características muito interessantes.

- $m \geq 2$ : é um tópico/combinação exclusivo, no qual as consequências têm um alcance não apenas na sua própria área de pesquisa. É provável que seja um tópico/combinação com efeitos de aplicação ou características únicas.

Os hot-topics conforme acima destacados foram anteriormente utilizados por Madruga e Silva (2008) na tentativa de analisar as publicações sobre o tema sustentabilidade no banco de dados da ISI Web of Science. Outro estudo que se apoia nesses índices foi realizado por Velter et al. (2010), que procuraram aprofundar os conhecimentos sobre as publicações na área da sustentabilidade e identificar quais tópicos relacionados a tal temática podem ser considerados hot-topics.

\subsection{Etapas e coleta de dados}

Para a coleta de dados utilizou-se a base de dados ISI Web of Science do índice de citações ISI Citation Indexes, publicado pela primeira vez em 1963, com dados de citações a partir de 1961 (GARFIELD, 1963). Essa base de dados oferece acesso direto ao fluxo de informações multidisciplinar sobre cerca de nove mil periódicos internacionais de alto fator de impacto para a pesquisa científica (THOMSON SCIENTIFIC, 2011).

Através dos instrumentos de análise apresentados por Madruga e Silva (2008) e Velter et al. (2010), promoveu-se um estudo no qual a coleta dos dados foi realizada através de uma busca simultânea dos termos desenvolvimento regional (regional development), desenvolvimento territorial (territorial development) 
e desenvolvimento local (local development) na base de periódicos internacionais ISI Web of Science. Considerados tais procedimentos, procedeu-se a coleta de dados no dia 28 de outubro de 2011.

A pesquisa dividiu-se em 4 etapas, descritas no Quadro 1. Na primeira etapa definiram-se os termos-chave para o levantamento de dados: regional development (desenvolvimento regional) ou territorial development (desenvolvimento territorial) ou local development (desenvolvimento local). Esses foram determinados devido à similaridade dos termos e divergências de conceitos e definições sobre os mesmos, o que tornaria a pesquisa de qualquer um deles em separado deficiente.

O delineamento do período de publicações foi 2001 a 2011. Ressalta-se, no entanto, que como o levantamento ocorreu em outubro de 2011 (impossibilitando que o ano fosse considerado em sua totalidade), para os cálculos dos índices h-b e m, foi estabelecido o período de 10,8 anos.

Quadro 1: Etapas da pesquisa.

\section{$1^{\text {a }}$ Etapa}

- Pesquisar os termos regional development ou territorial development ou local development, simultaneamente (2001 a 2011)

- Pesquisar: total de publicações, os autores com maior número de puclicações, áreas temáticas, título das fontes, ano das publicações, instituições, agências de financiamento, idiomas e países

- Identificar hot-topics

-1 ${ }^{\text {a }}$ busca na Web of Science:

- Tópicos selecionados: Actors, Business, Cluster, Competitiveness, Cooperation, Culture, Economy, Enterprises, Globalization, Governance, Innovation, Instittion, Managemento, Networks, Planning, Social Capital, Stakeholder, Strategy, Sustainability, Tourism.

\section{$2^{\text {a Etapa }}$}

- Identificar os hot-topics

- $2^{\mathrm{a}}$ busca na Web of Science:

- Combinação dos termos regional development ou territorial development ou local development com os tópicos da administração selecionados

\section{$3^{\text {a Etapa }}$}

- Hot-topics

- Índice $h-b$

- Índice $m$

- Hot-topics: Cluster, Globalization, Governance, Innovation, Institution, Networks, Strategy

\section{$4^{\text {a }}$ Etapa}

- Análise do número de publicações por autor e vezes que suas publicações foram citadas 
Essa primeira etapa permitiu que fossem levantas as seguintes informações a respeito das publicações encontradas: (a) total de publicações, (b) os autores com maior número de publicações, (c) áreas temáticas, (d) título das fontes, (e) ano das publicações, (f) instituições, (g) agências de financiamento, (h) idiomas e (i) países. Os resultados dessa etapa também possibilitaram a investigação dos principais artigos relacionados à temática central do estudo e, consequentemente, tornou-se possível enumerar vinte tópicos a serem combinados com os termos inicialmente pesquisados.

Na segunda etapa da pesquisa, foi feita uma busca no sistema, combinando cada um dos assuntos selecionados (possíveis hot-topics) aos termos regional development/territorial development/local development no mesmo período de tempo da primeira etapa (2001 a 2011). Em seguida, realizou-se a classificação das publicações e identificou-se os hot-topics.

Por fim, considerando o que ressalta Hirsch (2005), o número total de artigos publicados, embora meça a produtividade do autor, não mede a importância e/ou impacto de suas publicações, o que pode ser medido pelo número de citações que cada uma recebe, mensurado pelo h-index.

Para tanto, foi feita, na última etapa, uma análise da relação do número de publicações por autor e o número de vezes que o mesmo foi citado. Procedimento realizado com o propósito de averiguar se o total de publicações está diretamente relacionada à relevância da produção científica. Nesse momento da pesquisa, para cada combinação considerada hot-topic, foram selecionados os dez autores que mais publicaram e as dez publicações que foram mais citadas.

\section{APRESENTAÇÃO DOS RESULTADOS: O ESTUDO DO DESENVOLVIMENTO REGIONAL (2001 A 2011)}

No que diz respeito aos resultados do estudo, foram encontradas um total de 4.601 publicações, sendo que foram evidenciadas as 24 áreas que possuem o maior número de publicações abordando o desenvolvimento regional, conforme apresenta a Tabela 1.
Tabela 1: Áreas temáticas no estudo do desenvolvimento regional.

\begin{tabular}{|c|c|}
\hline Áreas temáticas & $\begin{array}{c}\text { N. }{ }^{\circ} \text { de } \\
\text { publicações }\end{array}$ \\
\hline Geography (Geografia) & 764 \\
\hline $\begin{array}{l}\text { Environmental Studies } \\
\text { (Estudos do Meio Ambiente) }\end{array}$ & 689 \\
\hline Economics (Economia) & 517 \\
\hline $\begin{array}{l}\text { Planning \& Development } \\
\text { (Planejamento \& Desenvolvimento) }\end{array}$ & 421 \\
\hline Urban Studies (Estudos Urbanos) & 383 \\
\hline Management (Gestão/Administração) & 291 \\
\hline Environmental Sciences (Ciências Ambientais) & 227 \\
\hline Business (Negócios) & 200 \\
\hline Operations Research Managent Science & 104 \\
\hline Public Administration (Administração Pública) & 101 \\
\hline Sociology (Sociologia) & 100 \\
\hline Ecology (Ecologia) & 95 \\
\hline Area Studies (Estudos Locais) & 86 \\
\hline $\begin{array}{l}\text { Engineering Environmental (Engenharia } \\
\text { Ambiental) }\end{array}$ & 76 \\
\hline Political Science (Ciências Políticas) & 75 \\
\hline Water Resources (Recursos Hidricos) & 68 \\
\hline Computer Science Information Systems & 60 \\
\hline $\begin{array}{l}\text { Geosciences Multidisciplinary } \\
\text { (Geociências Multidisciplinar) }\end{array}$ & 57 \\
\hline Agricultural Economics Policy & 52 \\
\hline Computer Sciences Interdisciplinary Applications & 51 \\
\hline Engineering Civil (Engenharia Civil) & 51 \\
\hline Computer Science Artificial Intelligence & 50 \\
\hline Energy Fuels (Energia) & 44 \\
\hline Business Finance (Negócios Financeiros) & 39 \\
\hline
\end{tabular}

Fonte: Elaborado a partir do banco de dados da ISI Web of Science.

Com relação aos autores com maior número de publicações, foram listados os 24 melhores ranqueados, conforme é demonstrado na Tabela 2

Quanto ao título das fontes, as 25 que mais publicaram no período investigado tiveram entre 151 e 19 publicações: Regional Studies (151), European Planning Studies (107), European Urban and Regional Studies (42), Environment and Planning C-government and Policy (41), Eure-revista Latinoamericana de Estudios Urbano 
Tabela 2: Autores com maior número de publicações sobre desenvolvimento regional.

\begin{tabular}{l|c}
\hline Autores & $\mathbf{N}^{{ }^{\circ}}$ de publicações \\
\hline WEI, Y. H. D. & 44 \\
\hline IEEE & 31 \\
\hline LIN, G. C. S. & 18 \\
\hline YEUNG, H. W. C. & 18 \\
\hline FRITSCH, M. & 15 \\
\hline HUDSON, R. & 14 \\
\hline SHEARMUR, R. & 13 \\
\hline SMITH, H. L. & 12 \\
\hline LUA & 11 \\
\hline NIJKAMP, P. & 11 \\
\hline BIRCH, K. & 10 \\
\hline BOEKEMA, F. & 10 \\
\hline HOU, R. Y. & 10 \\
\hline OECD & 10 \\
\hline PIRINEN, R. & 10 \\
\hline RAVBAR, M. & 10 \\
\hline ZHAO, P. J. & 10 \\
\hline BUCHTA, S. & 9 \\
\hline RODRIGUEZ, P. A. & 9 \\
\hline BELEZENTIS, A. & 8 \\
\hline HADJIMICHALIS, C. & 8 \\
\hline BATHEL, H. & 7 \\
\hline MACKINNON, D. & 7 \\
\hline SCOTT, A. J. & 39 \\
\hline FO .9 & \\
\hline
\end{tabular}

Fonte: Elaborado a partir do banco de dados da ISI Web of Science.

Regionales (40), Geoforum (40), Environment and Planning A (39), Tijdschrift Voor Economische en Sociale Geografie (38), Urban Studies (34), Entrepreneurship and Regional Development (32), Wit Transactions on Ecology and the Environment (31), Annals of Regional Science (30), Economic Geography (30), Eurasian Geography and Economics (29), Journal of Economic Geography (28), Papers in Regional Science (28), International Journal of Urban and Regional Research (27), Ekonomicky Casopis (25), Boletin de la Asociacion de Geografos Espanoles (23), Economic Science for Rural Development (23), Small Business Economics (23), Growth and Chance (22), Service Industries Journal (22), $3^{\text {rd }}$. Central Europeand Conference in Regional Science International Conference

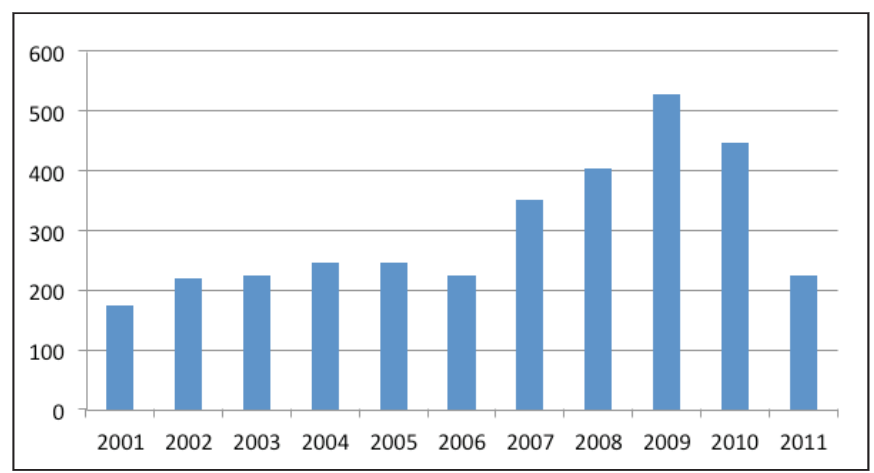

Fonte: Elaborado a partir do banco de dados da ISI Web of Science.

Gráfico 1: Número de publicações por ano, no período de 2001 a 2011.

Proceedings Young Sciences Articles (20), Progress in Human Geography (20) e Journal of Rural Studies (16).

Observando o histórico temporal das publicações envolvendo os termos regional development ou local development ou territorial development, constata-se um aumento gradativo a partir de 2007, tendo o ápice em 2009 (529 publicações) (Gráfico 1). Ressalta-se, novamente, que o ano de 2011 foi avaliado somente até o mês de outubro, o que pode influenciar no resultado final das análises envolvendo esse ano.

Quando levantadas as 25 instituições que mais publicaram sobre a temática desenvolvimento regional, observa-se que: 6 instituições são inglesas - University of Manchester (28), University Newcastle Upon Tyne (27), University Birmingham (18), University Hull (18), University Sheffield (16) e London's Global University (14); 5 instituições americanas - University Cambridge (21), University Wisconsin (19), University Utah (18), Cornell University (15) e Ohio State University (13); 2 chinesas Chinese Academy of Sciences (40), Wuhan University of Technology (16); 2 honcongonesas - University Hong Kong (21) e University Hong Kong (14); 2 escocesas - University Aberdeen (17) e University Stratchclyde (17); 1 sul-africana - University Utrecht (24); 1 singapuriana - National University of Singapore (23); 1 checa - Charles University of Prague (14); 1 holandesa - Erasmus University (14); 1 brasileira - Universidade de São Paulo (14); 1 lituana Vilnius Gediminas Technical University (14); 1 sueca - Lund University (13); e 1 italiana - University of Florence (13). 


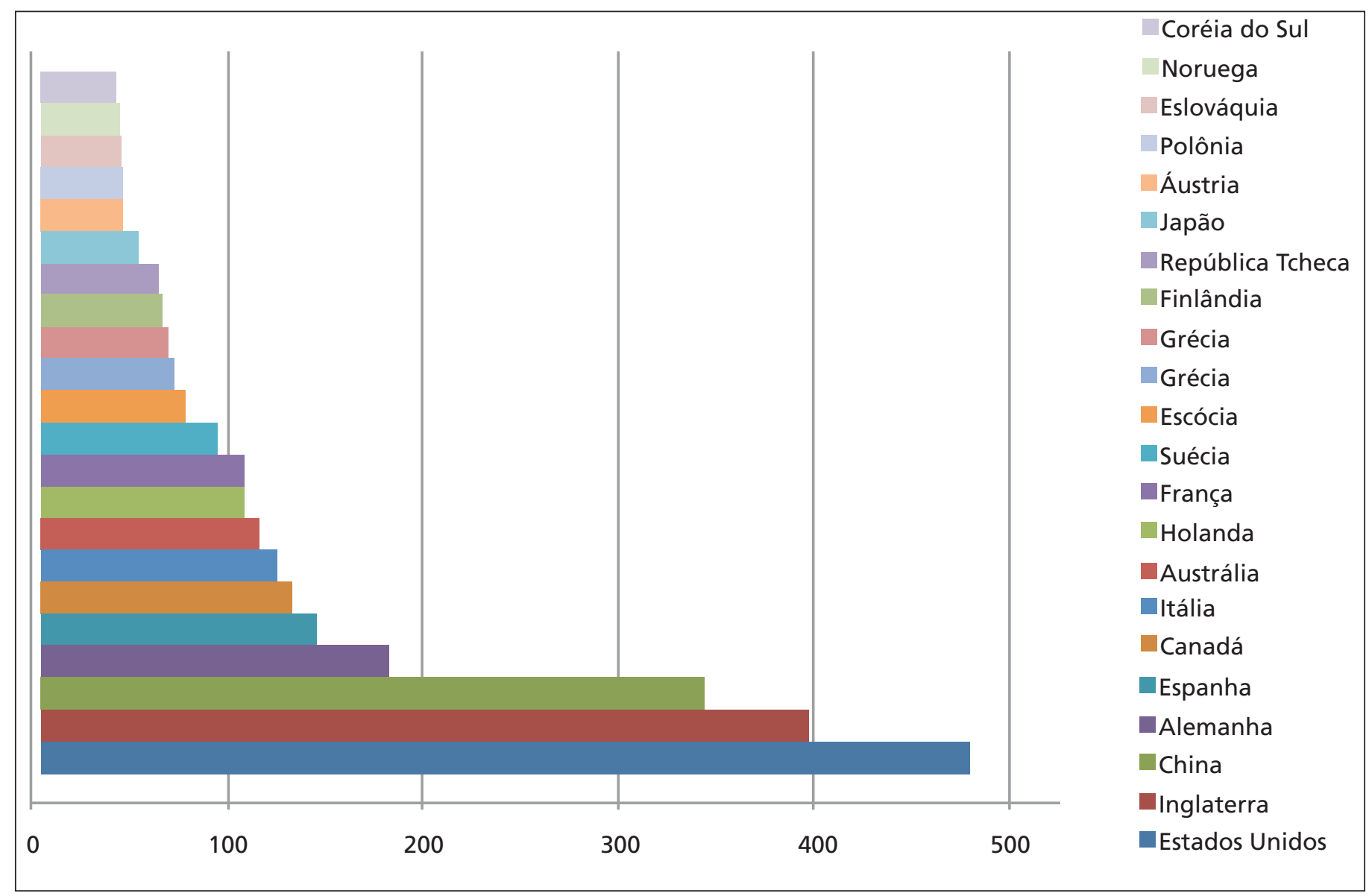

Fonte: Elaborado a partir do banco de dados da ISI Web of Science.

Gráfico 2: Publicações por países.

Em relação às agências financiadoras de trabaIhos que englobam o tema destacam-se a National Natural Science Foundation of China, com dezenove publicações; a European Commission, com sete publicações e a Chinese Academy os Sciences, com quatro publicações. Dentre as demais agências de financiamento, ressalta-se a presença do CNPq em quarto lugar, juntamente com a CAS, National Basic Research Program of China, Natural Sciences and Engineering Research Council of Canada e a $\mathrm{NIH}$, todas com três publicações. As demais instituições de financiamento que se destacaram tiveram no máximo duas publicações cada.

Quando observado o número de publicações por países de origem (Gráfico 2), os Estados Unidos lideram o ranking com 475 estudos. Em seguida, aparecem: a Inglaterra (393); a China (339); a Alemanha (178); a Espanha (141); o Canadá (129); a Itália
(121); a Austrália (112); a Holanda (107); a França (104); a Suécia (90); a Escócia (74); o Brasil (68); a Grécia (65); a Finlândia (62); a República Tcheca (60); o Japão (50); a Áustria (42); a Polônia (42); a Eslováquia (41); a Suíça (41); a Noruega (40) e a Coréia do Sul (39) (Gráfico 2).

A partir da comparação dos resultados entre as instituições que mais publicam e as agências financiadoras, pode-se notar que a maioria se encontra nos países de melhor colocação no ranking do Gráfico 2 . Complementando a análise, pode-se notar um predominio de publicações no idioma inglês, totalizando 89\% das publicações. Destacam-se também: o espanhol (98), o francês (57), o alemão (51) e o checo (22). Sobre as línguas, ressalta-se que o português aparece em $6^{\circ}$ lugar com 21 publicações. 
Tabela 3: Tópicos relacionados ao desenvolvimento regional, local e territorial selecionados para a pesquisa.

\begin{tabular}{l|c}
\hline Tópicos & Total de publicações \\
\hline Management (Gestão) & 680.034 \\
\hline Networks (Redes) & 643.542 \\
\hline Strategy (Estratégia) & 566.712 \\
\hline Culture (Cultura) & 430.907 \\
\hline Cluster & 322.083 \\
\hline Planning (Planejamento) & 234.326 \\
\hline Business (Negócios) & 95.458 \\
\hline Institution (Instituição) & 94.204 \\
\hline Economy (Economia) & 79.386 \\
\hline Innovation (Inovação) & 63.711 \\
\hline Enterprises (Empresas) & 50.578 \\
\hline Cooperation (Cooperação) & 45.176 \\
\hline Sustainability (Sustentabilidade) & 28.051 \\
\hline Governance (Governança) & 27.324 \\
\hline Actors (Atores) & 23.053 \\
\hline Globalization (Globalização) & 22.397 \\
\hline Stakeholder & 22.104 \\
\hline Tourism (Turismo) & 12.600 \\
\hline Competitiveness (Competitividade) & 12.037 \\
\hline Social capital (Capital social) & 5.417 \\
\hline
\end{tabular}

Fonte: Elaborado a partir do banco de dados da ISI Web of Science.

\subsection{O estudo do desenvolvimento regional e principais assuntos relacionados}

Nesta etapa da pesquisa, foram investigadas as publicações abordando o desenvolvimento regional, territorial e local relacionadas a assuntos relevantes, que comumente encontram-se associados a essas temáticas. Para que fosse possível determinar esses assuntos relevantes, realizou-se, previamente, uma pesquisa bibliográfica em livros, teses e artigos, como forma de obter insumos para construção de uma base teórica e possíveis tópicos relacionados ao tema.

Dessa forma, após a realização dessa pesquisa bibliográfica, a partir do conjunto de fichamentos, tornou-se possível reunir as ideias, construir um embasamento teórico e, consequentemente, propor os assuntos que seriam verificados como possíveis hot-topics. Salientados tais procedimentos, em ordem alfabética, os tópicos
Tabela 4: O estudo do desenvolvimento regional e seus hot-topics.

\begin{tabular}{|c|c|c|c|}
\hline Tópicos & $\begin{array}{c}\text { Total de } \\
\text { publicações }\end{array}$ & Índice h-b & Índice m \\
\hline $\begin{array}{l}\text { Economy } \\
\text { (Economia) }\end{array}$ & 732 & 33 & 3,05 \\
\hline $\begin{array}{l}\text { Strategy } \\
\text { (Estratégia) }\end{array}$ & 612 & 24 & 2,22 \\
\hline $\begin{array}{l}\text { Planning } \\
\text { (Planejamento) }\end{array}$ & 539 & 18 & 1,66 \\
\hline $\begin{array}{l}\text { Networks } \\
\text { (Redes) }\end{array}$ & 538 & 31 & 2,87 \\
\hline $\begin{array}{l}\text { Innovation } \\
\text { (Inovação) }\end{array}$ & 526 & 32 & 2,96 \\
\hline $\begin{array}{l}\text { Management } \\
\text { (Gestão) }\end{array}$ & 502 & 19 & 1,75 \\
\hline $\begin{array}{l}\text { Business } \\
\text { (Negócios) }\end{array}$ & 360 & 21 & 1,94 \\
\hline Cluster & 339 & 24 & 2,22 \\
\hline $\begin{array}{l}\text { Institution } \\
\text { (Instituição) }\end{array}$ & 278 & 22 & 2,03 \\
\hline $\begin{array}{l}\text { Governance } \\
\text { (Governança) }\end{array}$ & 271 & 22 & 2,03 \\
\hline $\begin{array}{l}\text { Globalization } \\
\text { (Globalização) }\end{array}$ & 244 & 24 & 2,22 \\
\hline $\begin{array}{l}\text { Actors } \\
\text { (Atores) }\end{array}$ & 230 & 20 & 1,85 \\
\hline $\begin{array}{l}\text { Enterprises } \\
\text { (Empresas) }\end{array}$ & 219 & 17 & 1,57 \\
\hline $\begin{array}{l}\text { Tourism } \\
\text { (Turismo) }\end{array}$ & 199 & 10 & 0,92 \\
\hline $\begin{array}{l}\text { Competitiveness } \\
\text { (Competitividade) }\end{array}$ & 184 & 16 & 1,48 \\
\hline $\begin{array}{l}\text { Sustainability } \\
\text { (Sustentabilidade) }\end{array}$ & 180 & 15 & 1,38 \\
\hline $\begin{array}{l}\text { Cooperation } \\
\text { (Cooperação) }\end{array}$ & 175 & 13 & 1,20 \\
\hline Stakeholder & 133 & 10 & 0,92 \\
\hline $\begin{array}{l}\text { Culture } \\
\text { (Cultura) }\end{array}$ & 128 & 12 & 1,20 \\
\hline $\begin{array}{l}\text { Social capital } \\
\text { (Capital social) }\end{array}$ & 84 & 13 & 1,20 \\
\hline
\end{tabular}

Fonte: Elaborado a partir do banco de dados da ISI Web of Science.

selecionados foram: Actors (Atores), Business (Negócios), Cluster, Competitiveness (Competitividade), Cooperation (Cooperação), Culture (Cultura), Economy (Economia), Enterprises (Empresas), Globalization (Globalização), 
Tabela 5: Relação entre os autores com mais publicações e publicações mais citadas.

\begin{tabular}{|c|c|c|}
\hline Tópicos & Autores com mais publicações & Publicações mais citadas \\
\hline $\begin{array}{l}\text { Economy } \\
\text { (Economia) }\end{array}$ & $\begin{array}{l}\text { Wei, Y. H. D. (16)1; Lin, G. C. S. (14); Birch, } \\
\text { K. (10); Balezentis, A. (8); Balezentis, T. (8); } \\
\text { Brauers, W. K. M. (8); Cumbers, A. (7); } \\
\text { Hudson, R. (7); Yeung, H. W. C. (7); } \\
\text { Coe, N. M. (6) }\end{array}$ & $\begin{array}{l}\text { 1.2 Coe, N. M.; Hess, M.; Yeung, H. W. C. et al. 'Globalizing' regional } \\
\text { development: a global production networks perspective. Transactions } \\
\text { of the Institute of British Geographers, v.29, n.4, p.468-484, dec. } 2004 \\
(168)^{3} \\
\text { 5. MacKinnon, D.; Cumbers, A.; Chapman, K. Learning, innovation } \\
\text { and regional development: a critical appraisal of recent debates. } \\
\text { Progress in Human Geography, v.26, n.3, p.293-311, jun. } 2002 \text { (125) } \\
\text { 10. Yeung, H. W. C.; Lin, G. C. S. Theorizing economic geographies } \\
\text { of Asia. Economic geography, v.79, n.2, p.107,128, apr. } 2003 \text { (74) }\end{array}$ \\
\hline $\begin{array}{l}\text { Innovation } \\
\text { (Inovação) }\end{array}$ & $\begin{array}{l}\text { Wei, Y. H. D. (17); Fristsch, M. (10); } \\
\text { Benneworth, P. (9); Smith, H. L. (9); } \\
\text { Harmaakorpi, V. (7); Barroso, I. C. (6); } \\
\text { MacKinnon, D. (6); Pirinen, R. (6); Shearmur, } \\
\text { R. (6); Bathelt, H. (6) }\end{array}$ & - \\
\hline $\begin{array}{l}\text { Networks } \\
\text { (Redes) }\end{array}$ & $\begin{array}{l}\text { Wei, Y. H. D. (17); Yeung, H. W. C. (15); } \\
\text { Pirinen, R. (10); Hudson, R. (9); Coe, N. M. } \\
\text { (8); Dicken, P. (7); IEEE (6); Hadjimichalis, C. } \\
\text { (6); Kalantaridis, C. (6); Djkema, G. P. J. (5) }\end{array}$ & $\begin{array}{l}\text { 1. Coe, N. M.; Hess, M.; Yeung, H. W. C. et al. 'Globalizing' regional } \\
\text { development: a global production networks perspective. Transactions } \\
\text { of the Institute of British Geographers, v.29, n.4, p.468-484, dec. } 2004 \\
\text { (168) } \\
\text { 4. Yeung, H. W. C. Rething relational economic geography. } \\
\text { Transactions of the Institute oh British Geographers, v.30, n.1, p.37-51, } \\
\text { mar. } 2005 \text { (126) } \\
\text { 6. Dicken, P.; Malmberg, A. Firms in territories: A relational perspective. } \\
\text { Economic Geography, v.77, n.4, p.345-363, oct. } 2001 \text { (123) }\end{array}$ \\
\hline $\begin{array}{l}\text { Strategy } \\
\text { (Estratégia) }\end{array}$ & $\begin{array}{l}\text { Lin, G. C. S. (10); IEEE (8); Baker, M. } \\
\text { (6); Balezentis, A. (6); Balezentis, T. (6); } \\
\text { Mauresan, M. (6); Wei, Y. H. D. (6); Zhao, P. } \\
\text { J. (6); Owusu, G. (5); Ozbek, O. (5) }\end{array}$ & $\begin{array}{l}\text { 5. Lin, G. C. S.; Ho, S. P. S. The state, land system, and land development } \\
\text { processes in contemporary China. Annals of the Association of American } \\
\text { Geographers, v.95, n.2, p.411-436, jun. } 2005 \text { (57) } \\
\text { 7. Lin, G. C. S. The growth and structural change of Chinese cities: a } \\
\text { contextual and geographic analysis. CITIES, v.19, n.5, p.299-316, oct. } \\
2002 \text { (53) }\end{array}$ \\
\hline Cluster & $\begin{array}{l}\text { Wei, Y. H. D. (18); Bathelt, H. (6); Boekema, } \\
\text { F. (6); Murphy, J. T. (6); Rovan, J. (6); } \\
\text { Benneworth (5); Hassink, R. (5); Lagendijk, } \\
\text { A. (5); Smith, H. L. (5); Zhou, Y. (5) }\end{array}$ & $\begin{array}{l}\text { 3. Hassink, R. How to unlock regional economies from path } \\
\text { dependency? From learning region to learning cluster. European } \\
\text { Planning Studies, v.13, n.4, p.521-535, jun. } 2005 \text { (56) } \\
\text { 7. Bathelt, H. Cluster relations in the media industry: Exploring the } \\
\text { 'distanced neighbour' paradox in Leipzig. Regional Studies, v.39, n.1, } \\
\text { p.105-127, feb. } 2005 \text { (37) }\end{array}$ \\
\hline $\begin{array}{l}\text { Globalization } \\
\text { (Globalização) }\end{array}$ & $\begin{array}{l}\text { Wei, Y. H. D. (38); Scott, A. J. (7); Yeung, H. } \\
\text { W. C. (7); Barton, J. R. (6); Lin, G. C. S. (6); } \\
\text { Hudson, R. (5); Zoomers, A. (5); Scott, A. J. } \\
\text { (5); Barroso, I. C. (4) }\end{array}$ & $\begin{array}{l}\text { 1. Coe, N. M.; Hess, M.; Yeung, H. W. C. et al. 'Globalizing' regional } \\
\text { development: a global production networks perspective. Transactions of } \\
\text { the Institute of British Geographers, v.29, n.4, p.468-484, dec. } 2004 \text { (168) } \\
\text { 4. Yeung, H. W. C. Rethinking relational economic geography. } \\
\text { Transactions of the Institute of British Geographers, v.30, n.1, p.37-51, } \\
\text { mar. } 2005 \text { (126) }\end{array}$ \\
\hline $\begin{array}{l}\text { Institution } \\
\text { (Instituição) }\end{array}$ & $\begin{array}{l}\text { Wei, Y. H. D. (20); MacKinnon, D. (5); } \\
\text { Bathelt, H. (4); Cumbers, A. (4); Murphy, J. } \\
\text { T. (4); Palascakova, D. (4); Ye, X. (4); Yeung, } \\
\text { H. W. C. (4); Helmsinf, A. H. J. (3); Phelps, N. } \\
\text { A. (3) }\end{array}$ & $\begin{array}{l}\text { 1. MacKinnon, D.; Cumbers, A.; Chapman, K. Learning, innovation } \\
\text { and regional development: a critical appraisal of recent debates. } \\
\text { Progress in Human Geography, v.26, n.3, p.293-311, jun. } 2002 \text { (125) } \\
\text { 7. Bathelt, H. Cluster relations in the media industry: Exploring the } \\
\text { 'distanced neighbour' paradox in Leipzig. Regional Studies, v.39, n.1, } \\
\text { p.105-127, feb. } 2005 \text { (37) }\end{array}$ \\
\hline $\begin{array}{l}\text { Governance } \\
\text { (Governança) }\end{array}$ & $\begin{array}{l}\text { Jonas, A. E. G, (7); Birch, K, (6); Coe, N. } \\
\text { M. (6); Dicken, P. (6); Abramovai, R. (4); } \\
\text { Coudele, E. (4); Fuller, C. (4); Maurel, M. C. } \\
\text { (4); Mauresan, M. (4); Rodriguez, P. A. (4) }\end{array}$ & $\begin{array}{l}\text { 4. Dicken, P.; Malmberg, A. Firms in territories: A relational perspective. } \\
\text { Economic Geography, v.77, n.4, p.345-363, oct. } 2001 \text { (123) }\end{array}$ \\
\hline
\end{tabular}


Governance (Governança), Innovation (Inovação), Institution (Instituição), Management (Gestão), Networks (Redes), Planning (Planejamento), Social Capital (Capital Social), Stakeholder, Strategy (Estratégia), Sustainability (Sustentabilidade) e Tourism (Turismo).

Apresentados os assuntos abordados, a Tabela 3 classifica os vinte tópicos conforme o número total de publicações sobre o tema, quando pesquisados individualmente.

Posteriormente, cada tópico apresentado pela Tabela 3 foi combinado com a pesquisa inicial dos termos regional development/local development/territorial development. Sendo calculado o total de publicações para cada combinação (tópico x desenvolvimento regional, territorial e local), o índice h-b e o índice m (Tabela 4). Os resultados foram listados seguindo o número total de publicações de cada combinação.

A partir do cálculo do índice h-b pode-se mensurar o desempenho dos tópicos/combinações pesquisados tendo por base o número total que estas tiveram (KELLY; JENNIONS, 2006).

No que diz respeito aos índices $h-b$ e $m$, partindo dos pressupostos levantados por Banks (2006), podese classificar como hot-topics, por possuírem o $\mathrm{m} \geq 2$, as combinações regional development/local development/territorial development com: economy $(3,05)$, innovation $(2,96)$, networks $(2,87)$, strategy $(2,22)$, cluster $(2,22)$, globalization $(2,22)$, institution $(2,03)$ e governance $(2,03)$. Já, as demais combinações, por apresentarem um $m>0,5$ podem ser consideradas como hot-topics emergentes.

Tendo por base as pesquisas realizadas na base de dados ISI Web of Science, foram selecionados, para as oito combinações consideradas hot-topics, os dez autores com maior número de publicações. Em seguida, foi investigado, dentre esses pesquisadores, quais aparecem como autores das dez publicações mais citadas para cada combinação (Tabela 5).

Na Tabela 5, relacionou-se o número de publicações por autores com o número de vezes que o mesmo e sua respectiva publicação foi citada. Através dos dados apresentados, pode-se observar que o número de publicações por autor não está diretamente relacionado com o impacto das mesmas, pois apenas os autores Coe (economy e networks), Yeung (economy, networks e globalization), Cumbers (economy e institution), Lin (economy e strategy), Dicken (networks e governance), Hassink (cluster), Bathelt (cluster e institution) e MacKinnon (institution) participam da lista das publicações mais citadas como pesquisadores.

Chama também a atenção o fato de que o autor Wei, mesmo sendo o líder do ranking dos autores nas categorias economy, innovation, networks, cluster, globalization e institution não possui nenhuma publicação entre as dez mais citadas em nenhum hot-topic.

\section{CONSIDERAÇÕES FINAIS}

O desenvolvimento regional constitui um tema que vem ganhando cada vez mais abrangência, tendo em vista que representa uma forma de proporcionar a competitividade de uma região em mercados globais e sistêmicos. Considerado esse aspecto, evidencia-se, também, um progressivo crescimento anual no que diz respeito ao total de publicações, especialmente a partir de 2007, chegando ao ápice no ano de 2009 (Gráfico 1).

Tais informações salientam a atualidade do tema proposto e que o mesmo vem adquirindo relevância e uma maior discussão em âmbito internacional, nas mais diversas áreas, em especial a geografia, o meio ambiente e a economia, conforme mostra a Tabela 1. No mais, nota-se que os resultados apresentados ressaltam o caráter multidisciplinar da temática (Tabela 3), o que pode apontar para uma diversidade de compreensões e falta de consenso no que diz respeito a abordagens, conceitos e definições.

A partir dos resultados, torna-se possível observar também que entre os autores com maior número de publicações, Wei é aquele que mais se destaca em relação aos demais com um total de 44 publicações (Tabela 2). Mas que esse número se faz pouco expressivo se comparado ao número total de publicações encontradas. Fato que pode apontar para uma possível dificuldade de se apontar um pesquisador ou um grupo como "referências obrigatórias" nas discussões sobre o tema. No entanto, embora se tenha notado tal fragilidade, pode-se considerar que os autores apontados podem ser vistos como alguns daqueles 
a partir dos quais se deve dar início à elaboração de referenciais teóricos sobre o tema.

Outro ponto relevante pode ser observado em relação às informações a respeito das 25 instituições que mais publicaram sobre a temática em epígrafe, pois podem auxiliar grupos de pesquisa e instituições a encontrar outros grupos internacionais que trabalham a mesma temática, como forma de estabelecer parcerias e promover o intercâmbio entre pesquisadores.

Com relação aos países com maior quantidade de publicações (Gráfico 2), nota-se que Estados Unidos, Inglaterra e China estão expressivamente a frente dos demais no ranking de publicações. Por outro lado, observa-se que o Brasil possui pequeno destaque no quadro internacional de publicações, estando na $13^{\text {a }}$ colocação. Tal dado mostra que o campo de estudos nacionais sobre o tema encontra-se ainda pouco explorado e evidencia a necessidade de realização de um maior número de estudos que possam discutir essa temática na realidade brasileira.

Como hot-topics identificou-se que economy (economia), strategy (estratégia), networks (redes), innovation (inovação), cluster, globalization (globalização), institution (instituição) e governance (governança) mostraram-se mais relevantes junto ao estudo do desenvolvimento regional.

Ressalta-se, no entanto, que tópicos como culture, que possui um número expressivo de publicações, quando relacionados com a temática proposta, o número total torna-se menos expressivo em relação às demais combinações. Por outro lado, percebe-se que outros tópicos que apresentam um número de publicações individualmente inferior (economy, innovation, governance e globalization), quando combinados, tornam-se mais expressivos em relação aos outros agrupamentos.
Além disso, considera-se que os hot-topics apontados apresentam categorias e temas que vêm sendo trabaIhados internacionalmente e que, pela relevância apontada, constituem pontos passíveis de serem abordados e aprofundados (em conjunto ou individualmente) por pesquisas futuras envolvendo o desenvolvimento regional.

Por fim, através das informações apresentadas pela Tabela 5, averiguou-se que o número de publicações por autor não está diretamente relacionado com o impacto das mesmas, pois apenas os autores Coe (economy e networks), Yeung (economy, networks e globalization), Cumbers (economy e institution), Lin (economy e strategy), Dicken (networks e governance), Hassink (cluster), Bathelt (cluster e institution) e MacKinnon (institution) participam da lista das publicações mais citadas e do ranking de pesquisadores com mais publicações (Tabela 2).

Chama a atenção, também, para o fato de que o autor Wei, mesmo sendo o líder do ranking dos autores nas categorias economy, innovation, networks, cluster, globalization e institution, não possui nenhuma publicação entre as dez mais citadas em nenhum hottopic. Essas informações mostram que não necessariamente aqueles que possuem o maior número de publicações possuem as mais relevantes (de impacto) para o conhecimento acadêmico em termos de número de vezes que foram citados, tendo suas contribuições reproduzidas em outros trabalhos.

Por fim, pode-se considerar como limitação do estudo o fato do mesmo ter sido realizado utilizandose apenas a base de periódicos internacionais ISI Web of Science. Por esta razão, sugere-se que estudos futuros desta natureza, possuam uma amplitude maior abrangendo outras bases de dados com o objetivo de complementar, comparar ou substituir os resultados encontrados nesta pesquisa. Outra sugestão é em relação ao período de anos a ser investigado, em que pode abranger uma faixa de tempo maior do que delimitada neste estudo. 


\section{REFERÊNCIAS}

ALBAGLI, S. Novos espaços de regulação na era da informação e do conhecimento. In: LASTRES, H. M. M.; ALBAGLI, S. (Org.). Informação e globalização na era do conhecimento. Rio de Janeiro: Campus, 1999. p. 290-313.

ARAÚJO, C. A. Bibliometria: evolução histórica e questões atuais. Em Questão, v.12, n.1, p.11-32, 2006.

BANKS, M. G. (2006). An Extension of the Hirsch index: indexing scientific topics and compounds. Disponível em: www.arxiv.org/abs/physics/0604216. Acesso em: 07 jul. 2010.

BECKER, D.; WITTMANN, M. (orgs). Desenvolvimento regional: abordagens interdisciplinares. Santa Cruz do Sul: EUNISC, 2003.

BASSAN, D. S.; SIEDENBERG, D. R. Desenvolver buscando a redução das desigualdades. In: BECKER, D. F; WITTMANN, M. L. Desenvolvimento regional: abordagens interdisciplinares. Santa Cruz do Sul: EDUNISC, 2008.

BOISIER, S. Em busca do esquivo desenvolvimento regional: entre a caixa-preta e o projeto político. Planejamento e Políticas Públicas, n.13, Brasília: IPEA, 1996.

BOISIER, S. Post-scriptum sobre desenvolvimento regional: modelos reais e modelos mentais. Planejamento e Políticas Públicas, n.9, Brasília: IPEA, 1999.

BUARQUE, S. C. Construindo o desenvolvimento local sustentável: metodologia de planejamento. Rio de Janeiro: Editora Garamond, 2004.

DALLABRIDA, V. R. O desenvo/vimento regional: a necessidade de novos paradigmas. ljuí: Ed. UNIJUÍ, 2000.

FIGUEIREDO, N. Tópicos modernos em bibliometria. Brasilia: Associação dos Bibliotecários do Distrito Federal, 1977.

FONSECA, E. N. Bibliometria: teoria e prática. São Paulo: Cultrix.Thomson Scientific, 2010.

GARFIELD, E. Science Citation Index. Science Citation Index 1961, v.1, 1963. Disponível em: http://garfield.library.upenn. edu/papers/80.pdf. Acesso em 28 jun. 2010.

GOBIERNO VASCO. Plan Forestal Vasco: 1994-2030. Dpto. Agricultura, 1994.
HIRSCH, J. E. An index to quantify an individual's scientific research output. Proceedings of the National Academy of Sciences of the United States of America (PNAS), California, v.102, n.46, p.16569-16572, 2005.

LORENZO, H. E.; FONSECA, S. A. A promoção do desenvolvimento local apoiada em redes de municípios: a experiência do Consórcio Intermunicipal Central Paulista. Interações, Campo Grande, v.9, n.1, p.55-63, jan.-jun. 2008.

MADRUGA, L. R. R. G.; SILVA, T. N. A localização das publicações e a identificação de tópicos quentes em sustentabilidade: uma primeira aproximação usando o Web of Science, XIV SIMPEP. Anais..., São Paulo, 2008.

MARTINS, S. R. O. Desenvolvimento local: questões conceituais e metodológicas. Revista Internacional de Desenvolvimento Local. v.3, n.5, p.51-59, set. 2002.

OLVEIRA, G. B. Uma discussão sobre o conceito de desenvolvimento. Revista FAE, Curitiba, v.5, n.2, p.37-48, maio-ago. 2002.

PIRES, M. S. Construção do modelo endógeno, sistêmico e distintivo de desenvo/vimento regional e a sua validação através da elaboração e da aplicação de uma metodologia ao caso do MERCOESTE. Tese (Doutorado em Engenharia de Produção). Universidade Federal de Santa Catarina. Florianópolis, 2001.

SACHS, I. Ecodesenvolvimento: crescer sem destruir. São Paulo: Vértice, 1986.

TAPIA, J. R. B. Desenvolvimento local, concentração social e governança: a experiência dos pactos territoriais na Itália. São Paulo em Perspectiva, v.19, n.1, p.132-139, jan.-mar. 2005.

Thomson Scientific. ISI Web of Science. Disponível em: http:// scientific.thomson.com/products/wos/. Acesso em: 20 abr. 2011.

VASCONCELOS, M. A.; GARCIA, M. E. Fundamentos de economia. São Paulo: Saraiva, 1998.

VÁZQUEZ BARQUERO, A. Desenvolvimento endógeno em tempos de globalização. Porto Alegre: Fundação de Economia e Estatística, 2001.

VELTER, A. N.; BATTISTELLA, L. F.; GROHMANN, M. Z.; CARPES, A. M. O estudo da sustentabilidade na administração: um levantamento dos "hot-topics" publicados na ultima década. Anais... XIII SemeAd - Seminários em Administração, 2010. 